\title{
An Online Adaptive Cooperation Scheme for Spectrum Sensing Based on a Second-Order Statistical Method
}

\author{
Serhan Yarkan, Behçet Uğur Töreyin, Khalid A. Qaraqe, Student Member, IEEE, and A. Enis Çetin, Fellow, IEEE
}

\begin{abstract}
Spectrum sensing is one of the most important features of cognitive radio (CR) systems. Although spectrum sensing can be performed by a single $\mathrm{CR}$, it is shown in the literature that cooperative techniques, including multiple CRs/sensors, improve the performance and reliability of spectrum sensing. Existing cooperation techniques usually assume a static communication scenario between the unknown source and sensors along with a fixed propagation environment class. In this paper, an online adaptive cooperation scheme is proposed for spectrum sensing to maintain the level of sensing reliability and performance under changing channel and environmental conditions. Each cooperating sensor analyzes second-order statistics of the received signal, which undergoes both correlated fast and slow fading. Autocorrelation estimation data from sensors are fused together by an adaptive weighted linear combination at the fusion center. Weight update operation is performed online through the use of orthogonal projection onto convex sets. Numerical results show that the performance of the proposed scheme is maintained for dynamically changing characteristics of the channel between an unknown source and sensors, even under different physical propagation environments. In addition, it is shown that the proposed cooperative scheme, which is based on second-order detectors, yields better results compared with the same fusion mechanism that is based on conventional energy detectors.
\end{abstract}

Index Terms-Adaptive data fusion (ADF), fast fading, mobility, online learning, projection onto convex sets (POCS), shadowing, spectrum sensing.

\section{INTRODUCTION}

$\mathbf{C}$ OGNITIVE radio (CR) systems, which are aware of their surroundings and have the capability of self-adaptation to dynamic environmental and channel conditions, have emerged

Manuscript received April 27, 2011; revised September 27, 2011; accepted November 11, 2011. Date of publication December 9, 2011; date of current version February 21, 2012. This work was supported by Qatar National Research Fund (QNRF) Grant through National Priority Research Program (NPRP) 08-101-2-025. QNRF is an initiative of the Qatar Foundation. The review of this paper was coordinated by Prof. O. B. Akan.

S. Yarkan is with the Department of Electrical and Electronics Engineering, İstanbul Commerce University, İstanbul 34840, Turkey (e-mail: syarkan@ ieee.org).

B. U. Töreyin is with the Department of Electronic and Communication Engineering, Çankaya University, Ankara 06530, Turkey (e-mail: toreyin@cankaya.edu.tr).

K. A. Qaraqe is with the Department of Electrical and Computer Engineering, Texas A\&M University at Qatar, Doha 23874, Qatar (e-mail: kqaraqe@tamu.edu).

A. E. Çetin is with the Department of Electrical and Electronics Engineering, Bilkent University, Ankara 06800, Turkey (e-mail: cetin@bilkent.edu.tr).

Color versions of one or more of the figures in this paper are available online at http://ieeexplore.ieee.org.

Digital Object Identifier 10.1109/TVT.2011.2179325 as a novel paradigm in wireless communications [1]. One of the most distinguished features of these systems is spectrum sensing for dynamic spectrum access. Dynamic spectrum access consists mainly of the following steps: 1) observing a specific portion of the radio frequency (rf) spectrum steadily; 2) deciding whether the portion of interest is occupied or not; and 3) exploiting the opportunities in such a way that no harm is done to primary users. From the perspective of both 2) and 3), it can be said that agility and accuracy are the two prominent requirements for CRs in spectrum sensing since CRs need to be accurate in their decisions about whether there is spectrum opportunity. Furthermore, once an opportunity (or a licensed user) emerges, they need to be very agile to take appropriate actions (e.g., exploiting a white hole or vacating the band due to an emerging primary user) in a timely manner.

Accuracy in spectrum sensing is generally inversely proportional to the complexity of CR systems. However, an increase in complexity implies a decrease in agility, which constitutes a critical design tradeoff [2], [3]. Therefore, most of the time, it is difficult to attain both utmost agility and high accuracy in receiver design simultaneously. Particularly in spectrum sensing, such tradeoffs lead to various methods described in the literature, which range from those that give priority to agility to those that give priority to accuracy. As will be discussed subsequently, all of these methods are somehow related to the amount of knowledge in hand before spectrum sensing operations take place.

Spectrum sensing can generally be considered under the absence or presence of a priori knowledge of the signal to be detected. In case there is no knowledge about the signal, it is shown that the optimal detector is an "energy detector," which is a noncoherent receiver measuring solely the energy of the received signal over a specific period of time for a specific band [4]. The energy detector has very critical drawbacks such as being prone to uncertainties in noise variance [5], exhibiting degraded performance for low signal-to-noise ratio (SNR) values [6], and performing unsatisfactorily, particularly in detecting spread spectrum signals [7]. As opposed to the no-knowledge case, when the signal itself is completely known, the optimal detection in a stationary Gaussian noise is a matched filter (with a threshold comparison) [8]. Note that having complete knowledge of a signal includes a very long and detailed set of parameters, some of which are signaling bandwidth, operating frequency, modulation type and order, pulse shape, frame/burst format, and so on. As can be seen from this list, the optimal 
solution under a certain assumptions comes at the expense of obtaining a very broad knowledge of the signal. It is obvious that such a broad knowledge might not always be available. Instead of having complete knowledge, partial knowledge of the signal might be available, such as the signal to be detected being digitally modulated with a certain (known) symbol rate. In such scenarios, more complex architectures, which still include matched filters, can safely be used in an optimal sense [9]. Another approach in partial knowledge scenarios is known as "waveform-based sensing." In waveform-based sensing, a set of known characteristics of the signal whose absence/presence is to be detected is searched across the received signal with the use of specially constructed templates [6], [10]. The main drawback of waveform-based sensing methods is that they still need to rely on the knowledge of some distinct characteristics of the transmitted signal although the knowledge is partial. Slightly different from waveform-based sensing approach, cyclostationarity-based methods strive to exploit the inherent periodicity in the statistics of a signal such as its autocorrelation [11], [12]. This way, instead of focusing directly on the knowledge of the signal itself, an indirect approach is adopted by investigating its inherent statistical features. Particularly under the "signal interception" umbrella, more comprehensive and unifying studies based on several inherent statistical characteristics of very broad classes of signals can also be found in the literature [13]. Despite the fact that it is a very powerful method, cyclostationarity-based methods might be relatively more computationally complex. Similar to cyclostationarity-based methods, correlation-based approaches can also be applied to spectrum sensing problems [14]-[16]. However, spatial correlation of shadowing needs to be taken into account since it changes the statistical characteristics of signals and affects the performance [16]. Apart from these, there are also some studies that consider multilevel sensing. For instance, in radio identification-based sensing, some approaches extract features of the signal first, and then, these features are fed to another level to identify the absence/presence of a primary user. Another approach in multilevel sensing is to take into account both local and global decisions along with some sort of decision rule. For a very comprehensive list of studies, including various other methods on spectrum sensing available in the literature, see [17]-[22] and references therein.

Up until this point, spectrum sensing methods are reviewed from the perspective of a single CR or sensor. ${ }^{1}$ However, there are scenarios in which multiple sensors might be used in spectrum sensing. When multiple sensors are involved in spectrum sensing, several concerns arise. (C.i) First of all, many sensors imply many input data; therefore, a decision/data fusion is essential to come up with a single decision on the absence/presence of an unknown source. Decision fusion forces one to contemplate a fusion architecture. (C.ii) Second, once a plausible architecture is proposed, a mechanism needs to be established so that the deficiency in observations of each individual sensor stemming from receiver uncertainty, fading, and shadowing will be overcome. Hence, cooperative spectrum

\footnotetext{
${ }^{1}$ From this point on the terms "CR" and "sensor" are used interchangeably.
}

sensing schemes are proposed by taking into account both (C.i) and (C.ii) to improve the sensing performance through the use of spatial diversity [19], [23]-[26]. Although there is vast and ever-increasing studies in the literature on cooperative spectrum sensing (see [19], [26], and references therein), most of the existing methods are developed based on a static communication scenario between the unknown source and the sensors along with fixed rf propagation environment classes and characteristics. However, it would be too optimistic to state that these cooperative techniques, with their non-adaptive nature, can survive in practice under dynamically shifting channel and environmental conditions.

Online learning approaches are powerful tools for problems where drifts in concepts take place. It is important to observe that many CR problems accommodate dynamic characteristics in many aspects. These dynamic characteristics cause drifts in paradigms. For instance, mobility not only causes correlated fast fading but also leads to changes in propagation environment class or characteristics, such as moving from urban to suburban area. Since such drastic changes need to be taken into account in spectrum sensing, an online learning approach seems very promising, particularly from the perspective of practical CR systems. Therefore, in this paper, an adaptive data fusion (ADF) scheme, which exploits the inherent dynamics of the sensing problem by adapting the weight of the contribution from each spectrum sensor in an online manner, is proposed for cooperative spectrum sensing. Each sensor carries out a set of operations based on second-order statistical characteristics of the received unknown signal, which is assumed to be emitted by a mobile source. Considering the fact that shadowing process changes slowly compared with fast-fading process, detectors aim to separate the statistics of these processes from the received signal by employing a low-pass filter followed by a logarithmic detector and investigating the second-order statistics of the output. Then, the output value of each sensor is sent to the fusion center for their corresponding weights to be updated online based on the ADF scheme through the use of orthogonal projections onto convex sets (POCS). In this regard, the contributions of this paper can be summarized as follows. (C.I) All of the main propagation mechanisms in the physical layer, such as correlated fast and slow fading, are taken into account in each spectrum sensor. (C.II) A second-order detector is employed in sensors that can decide individually on the absence/presence of the unknown signal. (C.III) An adaptive cooperation scheme is proposed to maintain the reliability of the spectrum sensing system online by tracking the dynamics in the channel and the propagation environment via the output of the second-order detectors. Note that the general framework of a POCS-based online adaptive decision fusion scheme was introduced in [27] for machine vision application. This general framework was then applied for a cooperative spectrum sensing problem where each sensor utilizes an energy-based spectrum sensing approach in [28] and [29]. Different from previous studies, in this paper, the fusion mechanism exploits the bounded input generated by second-order detectors.

The organization of this paper is as follows. The system model and the statement of the problem are presented in Section II. In Section III, the proposed online adaptation and 


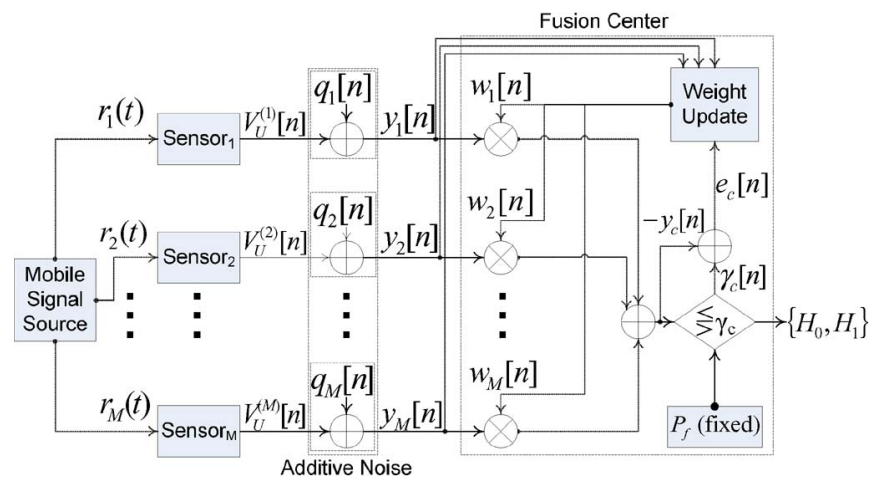

Fig. 1. Block diagram for the proposed method and for the second-order detector embedded in spectrum sensors.

data fusion method is described. Numerical results and discussions are presented in Section IV. Finally, conclusions are drawn in the last section.

\section{System Model and Statement of the Problem}

Let a fixed immobile spectrum sensor network be composed of $M$ sensors, possibly situated at $M$ geographically different locations. Each sensor, for example, Sensor ${ }_{i}$, where $i=$ $1, \ldots, M$, carries out a sequence of operations upon receiving a signal $r_{i}(\cdot)$ coming from a single unknown radio source. Next, each sensor yields an output value $V_{U}^{(i)}[n]$ at a discrete time index, for example, $n$, and sends it over a broadcast channel to a fusion center. In the final step, the fusion center takes all the discrete inputs collected over the broadcast channel, for example, $\left\{y_{i}[n]\right\}$, and combines them in such a way that at the end a binary decision is performed based on a threshold under certain conditions such as a fixed probability of false alarm. It is worth mentioning here that spectrum assignment is assumed to be already established before the system runs and maintained throughout the entire period of operation. Broadcast fusion channel is assumed to function of simplex mode, which solely conveys sensor output to the fusion center. In case a new spectrum assignment is required, then the new assignment information is assumed to be dispatched to the sensors through the use of a reserved control channel. An outline of the system model described here is depicted in Fig. 1.

In light of the system model given above, the problem can be stated as follows: identifying the absence/presence of an unknown narrow band radio source by analyzing it with $M$ different, independent, and individual sensors and then coming up with a binary decision on the absence/presence of the unknown source by fusing the output of the broadcast channel carrying sensor output values under a specific probability of false alarm values.

Stemming from the fact that the system model considered in this paper functions in multiple steps as discussed above, it is useful to contemplate each step individually. However, to formalize the overall behavior of the system model, let the following be given with regard to the input and output of the entire system considered in the following:

$$
r_{i}(t)=\left\{\begin{array}{rr}
n_{i}(t), & H_{0} \\
x_{i}(t)+n_{i}(t), & H_{1}
\end{array}\right.
$$

where $x_{i}(t)$ is the output of the channel (including slow and fast fading) between the unknown source and Sensor $_{i}$, and $n_{i}(t)$ is the ambient noise at the antenna of $\operatorname{Sensor}_{i}$. In (1), $H_{0}$ corresponds to the case where the unknown source is absent, whereas $H_{1}$ corresponds to the case where the unknown source is present. It is important to keep in mind that the absence/presence of the unknown source is universal for each and every Sensor $_{i}$ since the system model described above assumes that there is a single unknown radio source. However, the system model given above is not limited to identifying the absence/presence of a single unknown source. To see this, one can imagine that there is more than one unknown active source. It is clear that there is no difference between a single active unknown source and multiple active unknown sources since there is at least one unknown activity in either case. On the contrary, the absence of unknown source requires that there is absolutely no active unknown source. Since the difference between the absence and presence of an unknown source is solely a single active unknown source, this paper focuses on a single unknown source scenario.

\section{A. Channel Characteristics Between Sensors and The Unknown Source}

By adopting complex baseband representation, for each Sensor ${ }_{i}$, the noise process $n_{i}(t)$ is assumed to be of complex additive white Gaussian noise (AWGN) form with $\mathcal{C N}\left(0, \sigma_{N}^{2}\right)$ as $n_{i}(t)=n_{i}^{I}(t)+j n_{i}^{Q}(t)$, where both $n_{i}^{I}(t)$ and $n_{i}^{Q}(t)$ are $\mathcal{N}\left(0, \sigma_{N}^{2} / 2\right)$ and $j=\sqrt{-1}$. On the other hand, $\left\{x_{i}(t)\right\}$ are assumed to be narrowband signals, where the delay spread of each channel (the channel between the unknown source and Sensor $_{i}$ ) is relatively small compared with the inverse bandwidth of the channel of interest. Therefore, under the narrowband assumption for Sensor ${ }_{i}$, the unknown signal $x_{i}(t)$ can be modeled by decomposing it into the following form:

$$
x_{i}(t)=m_{i}(t) s_{i}(t) a(t)
$$

where $m_{i}(t)=h_{i}(t) e^{j \theta_{i}(t)}$ represents the complex fading channel process whose amplitude and phase are denoted with $h_{i}(t)$ and $\theta_{i}(t)$, respectively; $s_{i}(t)$ denotes the real-valued slowfading process including the combined effects of both distancedependent path loss and shadowing; and $a(t)$ is the unknown baseband signal. In addition, all three processes in (2) are assumed to be independent of each other and of the noise process $n_{i}(t)$.

Note that for the sake of notational convenience, the index ${ }_{i}$ representing the $i$ th sensor will be dropped, and all analyses will hereafter be carried out for a single, generic sensor, unless otherwise stated.

The complex fading channel process $m(t)$ is composed of multiple rays (sometimes referred to as paths) arriving at the receiver antenna and causes rapid fluctuations in the power level of the received signal with respect to very small displacements on the order of a couple of wavelengths of the transmission. Complex fading channel process is mainly characterized by the distribution of its fading amplitude $h(t)=|m(t)|$. In the literature, some of the frequently used fading amplitude 
distributions are Rayleigh, Rice, and Nakagami- $m$ distributions. In addition to amplitude distribution, Doppler spectrum of the fading channel process is also important in characterizing the complex fading process [30]. Two of the frequently used models in the literature for Doppler spectrum are Jakes' and Gaussian Doppler spectrum.

It is known that both path loss and shadowing change more slowly compared with the fast-fading process $m(t)$. Therefore, there is no harm in modeling both path loss and shadowing with a single process as follows [31], [32]:

$$
s(t)=\exp \left(\frac{1}{2} \mu(t)+\frac{\sigma_{G}}{2} g(t)\right)
$$

where $\mu(t) / 2$ denotes mean, $\sigma_{G} / 2$ is the standard deviation of log-normal shadowing, and $g(t)$ is a real-valued unit normal process $\mathcal{N}(0,1)$. Moreover, the experimental studies present in the literature for shadowing process $g(t)$ show that shadowing correlation can be approximated by the following model [33]:

$$
R_{g}(\tau)=E\{g(t) g(t+\tau)\}=\exp \left(-\frac{v|\tau 1|}{d_{\rho}}\right) .
$$

Note also that there are some other studies in the literature related to shadowing models, such as static and dynamic shadowing [34]. Yet, the model defined by both (3) and (4) is adopted due to the following two reasons: (R.1) It is clear that due to the mathematical tractability of both (3) and (4), the analysis will be simpler. (R.2) Furthermore, such a sharp (exponential) decay yields pessimistic results in terms of shadowing correlation, which provides some sort of upper bound for the problem considered. Having said this, as will be shown subsequently, it is important to mention that the proposed method is independent of any sort of shadowing correlation model.

Finally, without loss of generality, it is assumed that displacement of the unknown source within the duration of operation is negligibly small compared with the distance between the unknown signal source and the sensors. Therefore, the impact of $\mu(t)$ can be neglected so that $s(t)$ is assumed to solely include the impact of the shadowing process.

\section{B. Channel Characteristics Between Fusion Center and Sensors}

The channel between the fusion center and the sensors is called "broadcast fusion channel." As opposed to the channel between sensors and an unknown source, the broadcast fusion channel is considered to be of discrete form for the sake of notational convenience. However, this assumption does not affect the essence of the method proposed at the fusion center.

Since both sensors and the fusion center (and even the objects in between) are assumed to be immobile, one can assume that the broadcast channel gains do not change in time. Because there is ambient noise at the fusion center, the broadcast fusion channel can be considered to be an AWGN channel. Note that such conditions are valid for transmissions through a guided media, such as a direct cable connection between the fusion center and the sensors. In addition, fixed scenarios such as rooftop-to-rooftop communications with the presence of a very strong line of sight (LOS) can be considered to be AWGN channel [35], [36]. Such scenarios are nothing but special versions of a more generalized scheme where there is a stationary transmitter and a fixed receiver that is equipped with an antenna of high directivity. In such generalized schemes, the channel between the transmitter and the receiver falls into the AWGN channel category because the directivity of the receiver antenna can be adjusted in such a way that either direction of the strong LOS or the strongest path is aimed at [37] and [38]. Furthermore, it is known that under fairly general conditions, fading channels can be transformed into AWGN form by increasing the number of diversity branches [39]. In this regard, the set of signals reached at the fusion center at the $n$th discrete time instant can be modeled as a Gaussian channel with zero-mean noise $q_{i}$ and with variance $\delta=\left[\delta_{1}^{2}, \delta_{2}^{2}, \ldots, \delta_{M}^{2}\right]^{T}$ for the sake of an easier analysis

$$
\mathbf{y}=\mathbf{V}_{\mathbf{U}}+\mathbf{q}
$$

with $\mathbf{y}=\left[y_{1}[n], \ldots, y_{M}[n]\right]^{T}, \quad \mathbf{V}_{\mathbf{U}}=\left[V_{U}^{(1)}[n], \ldots, V_{U}^{(M)}[n]\right]^{T}$, and $\mathbf{q}=\left[q_{1}[n], \ldots, q_{M}[n]\right]^{T}$, where $(\cdot)^{T}$ denotes the transpose operation.

\section{Proposed Method}

In this section, the details of how sensors operate to come up with their output values, namely, $V_{U}^{(i)}[n]$, will be discussed. Following that, the analysis of the fusion center will be investigated.

\section{A. Sensors}

In Section II, it is stated that statistics of the shadowing and fast-fading processes evolve in different scales on the spatial domain. This implies that the shadowing process is not expected to vary within relatively short displacements, such as in a couple of wavelengths of the transmission. Keeping this in mind, first consider passing the received signal through a low-pass filter whose (normalized) impulse response is given by

$$
w(t)=\frac{1}{2 T_{A}}\left(\operatorname{sgn}\left(t+\frac{T_{A}}{2}\right)-\operatorname{sgn}\left(t-\frac{T_{A}}{2}\right)\right)
$$

where $\operatorname{sgn}(\cdot)$ is the signum (or sign) function, and $T_{A}$ denotes the effective averaging duration. Now, consider the hypothesis $H_{1}$ since it includes both noise and unknown signal terms. If $r(t)$ is passed through the low-pass filter under the hypothesis $H_{1}$, then, in light of both (2) and (6) and after some mathematical manipulations [16]

$$
\begin{aligned}
z(t) & =s(t) \underbrace{\int_{t-T_{A} / 2}^{t+T_{A} / 2} w(t-\tau) m(\tau) a(\tau) d \tau}_{M_{F}(t)}+N_{F}(t) \\
& =s(t) M_{F}(t)+N_{F}(t)
\end{aligned}
$$


is obtained, where $N_{F}(t)$ denotes the low-pass-filtered white Gaussian noise, and $T_{A}$ is assumed to be so short that shadowing does not change within. ${ }^{2}$

Next, the natural logarithm operator is applied to the absolute square of $z(t)$ to reveal the impact of the shadowing process, which reads

$$
\ln (Z(t))=\ln \left(s^{2}(t)\left|M_{F}(t)\right|^{2}\right)+\ln \left(\frac{Z(t)}{\left|s(t) M_{F}(t)\right|^{2}}\right) .
$$

With the aid of both (3) and (8)

$$
\ln (Z(t))=\sigma_{G} g(t)+\underbrace{\ln \left(\left|M_{F}(t)\right|^{2}\right)+\ln \left(\frac{Z(t)}{\left|s(t) M_{F}(t)\right|^{2}}\right)}_{L(t)}
$$

is obtained by neglecting the impact of distance-dependent path loss.

It is clear that the autocorrelation of (9) will include the shadowing correlation via $g(t)$. Therefore, the unbiased estimate of autocorrelation of (9) is found to be

$$
R_{\ln (Z)}(\tau)=\sigma_{G}^{2} e^{-v|\tau 1| / d_{\rho}}+R_{L}(\tau)+R_{g L}(\tau)+R_{L g}(\tau)
$$

Although the impact of shadowing is clear in (10), it is difficult to come up with a definitive statement with regard to the presence of an unknown signal. This mainly stems from the following two reasons: First, remnants of the low-pass filtering operation cannot be removed completely. Second, the autocorrelation estimates are biased with the mean of $\ln (Z(t))$. In addition to these two, one should also keep in mind that finite support leads to drastic fluctuations in autocorrelation estimates and renders the entire decision process difficult. However, as will be shown subsequently, all these issues can be remedied by investigating the noise-only process through steps (7)-(10). In the following parts, (10) will be discussed further in light of the observations for the noise-only case. First, the following needs to be given.

Proposition 1: Under the hypothesis $H_{0}$ along with the ideal conditions, such as $T \rightarrow \infty, T_{A}=0$, and unit variance noise, the normalized output of the correlator of any Sensor ${ }_{i}$ converges the constant $\Phi^{\prime}=\gamma^{2} / \gamma^{2}+\pi^{2} / 6$, where $\gamma$ is the Euler-Mascheroni (or sometimes referred to solely as Euler's) constant.

Proof: See the Appendix.

Note that $T_{A}=0$ implies no low-pass filtering operation. It is clear that a low-pass filter applied prior to the logarithmic detector will not alter the convergent behavior of $\Phi^{\prime}$. However, it will change the value of $\Phi^{\prime}$ to a new constant, for example, $\Phi_{T_{A}}^{\prime \prime}$. Since there is no closed form, by relaxing some of the conditions imposed in the Appendix, $\Phi_{T_{A}}^{\prime \prime}$ can be approximated with $\Phi_{T_{A}}^{\prime \prime}=k^{2} \gamma^{2} / k^{2} \gamma^{2}+\pi^{2} / 6$ as an extension to (42). Here, $k \in \mathbb{Z}^{+}$denotes the number of samples that are

\footnotetext{
${ }^{2} \mathrm{~A}$ brief discussion regarding to what extent $T_{A}$ can be considered to be short is given in Section IV for both practical scenarios and general cases.
}

taken $1 / f_{s}$ s apart, and $f_{s}$ is the sampling frequency of the receiver.

Since finite support leads to fluctuations around $\Phi_{T_{A}}^{\prime \prime}$ at larger $\tau$, the autocorrelation estimates in (10) are fed into the following unbiased estimator prior to the decision step:

$$
V_{U}=\frac{1}{U} \int_{T_{A}}^{T_{A}+U} R_{\ln (Z)}(\tau) d \tau
$$

where $U$ denotes the effective integration time. It is clear that when $T_{A}=0^{+}$and $U \rightarrow \infty$, then $V_{U} \rightarrow \Phi^{\prime}$.

Analysis of the noise-only case reveals that a drastic drop is anticipated at the output of the correlator for $H_{0}$. On the other hand, for $x(t) \neq 0$, although a drop will still be observed, it will not be as drastic as that in the noise-only case. ${ }^{3}$ Therefore, one can conclude that

$$
\Phi_{T_{A}}^{\prime \prime}<V_{U}
$$

always holds. Moreover, as shown in the Appendix, $\Phi_{T_{A}}^{\prime \prime}$ implies that no such measurement is required to determine a specific threshold.

Considering the practical aspects, $T_{A}$ can be selected to be the lowest nonzero value that is possible at the receiver. This way, the assumption regarding the invariance of shadowing is still maintained. On the contrary, $U$ should be chosen as large as possible to obtain better estimates of $V_{U}$ in (11). Therefore, one can conclude that $U$ is actually bounded by the memory (or buffer) capacity of the receiver.

In the sequel, it is critical to emphasize that each and every sensor is actually able to make a decision on the absence/presence of an unknown source through the use of $V_{U}$, as explained in [16]. However, in this paper, the scenario in which decisions are made by individual sensors are not considered. Therefore, it is assumed that each Sensor ${ }_{i}$ sends the output of its correlator at time instant $n$, namely, $V_{U}^{(i)}[n]$, to the fusion center through the use of a broadcast channel.

\section{B. Fusion Center}

Sensors process received signals in accordance with the second-order statistical method whose details are given in Section III-A. Here, it is important to recall that the output of each sensor can be considered to be sent at discrete time instants. Sensor outputs are transmitted over a very narrowband channel, namely, the broadcast fusion channel, to an immobile "fusion center." In this regard, based also on the practical layout scenarios introduced in Section II-B, such as rooftop-to-rooftop (or through the use of a guided media) communications, the set of signals reached at the fusion center at the $n$th discrete time instant is assumed to be modeled as expressed in (5). At the fusion center, where the ADF is realized online, a decision

\footnotetext{
${ }^{3}$ This is very critical because of the reason (R.2) stated in Section II Considering the fact that the proposed method is independent of any specific correlation model for shadowing, $\Phi_{T_{A}}^{\prime \prime}$ constitutes the lower bound for the problem considered here.
} 
is made through the use of a global test statistic $y_{c}[n]$ that is computed from $y_{i}[n]$ as follows:

$$
y_{c}[n] \gtreqless \mathcal{H}_{1} \gamma_{0}[n]
$$

where

$$
\begin{aligned}
& y_{c}[n]=\sum_{i=1}^{M} y_{i}[n] w_{i}[n]=\mathbf{y}^{T}[n] \mathbf{w}[n] \\
& \mathbf{w}[n]=\left[w_{1}[n], \ldots, w_{M}[n]\right]^{T}, \quad w_{i}[n] \geq 0 .
\end{aligned}
$$

The weight vector $\mathbf{w}[n]$ corresponding to spectrum sensors is updated to maintain the same $P_{f}$ under dynamically changing channel and propagation environment characteristics. Physically, weight vectors are affected by mobility, shadowing, and type of the propagation environment, such as urban or suburban. Dynamical changes in the environment and channel directly affect the output of the sensors $V_{U}$ 's, which consequently affect the weights at the fusion center.

Recall from Section III-A that under hypothesis $H_{0}$, the output of the correlator converges to a constant $\left(V_{U} \rightarrow \Phi^{\prime}\right)$, whereas it yields always greater values, for example, $\Phi^{+}$, than the constant $\Phi^{\prime}$ for the same settings $\left(T_{A}=0\right)$ under the hypothesis $H_{1}$. As will also be shown in Section IV, the output of correlators under the hypothesis $H_{1}$ can be assumed to be constant as well with the aid of the unbiased estimator given in (11). It is a key observation that the constant $\Phi^{\prime}$ is universal for all sorts of environmental classes such as urban and suburban, whereas $\Phi^{+}$changes from one environment to another. Although $\Phi^{+}$changes depending on the environment, within the decision process assuming that environmental class does not change, $\Phi^{+}$is considered to be a constant. Therefore, it is clear that $\mathbf{y}$ in (5) is actually another normal random variable because both $\Phi^{\prime}$ and $\Phi^{+}$are constants. Bearing in mind that the linear combination of normal random variables yields another normal random variable (with probably different mean and standard deviation), the expected value of the weighted combination for the unit variance noise at the sensor input at any time instant $n$, namely, $y_{c}[n]$, is given by

$$
E\left\{y_{c}[n]\right\}= \begin{cases}\Phi^{\prime}\|\mathbf{w}[n]\|_{1}, & \text { if } H_{0} \\ \Phi^{+}\|\mathbf{w}[n]\|_{1}, & \text { if } H_{1}\end{cases}
$$

where $\Phi^{\prime}<\Phi^{+}$, and $\|\cdot\|$ is the $\mathbf{L}_{\mathbf{1}}$ norm since $\left\{w_{i}\right\}$ 's are defined to be nonnegative in (15). Variance of $y_{c}[n]$ can then be calculated via (16) a

$E\left\{\left(y_{c}[n]-E\left\{y_{c}[n]\right\}\right)^{2}\right\}=\mathbf{w}^{T}[n] \operatorname{Cov}_{\mathbf{q}[\mathbf{n}]} \mathbf{w}[n]=\sum_{i=1}^{M}\left(w_{i} \delta_{i}\right)^{2}$

for both $H_{0}$ and $H_{1}$, where $\operatorname{Cov}_{\mathbf{q}[\mathbf{n}]}$ denotes the covariance matrix of $\mathbf{q}[n]$. The key observation here is that the outputs of sensors are actually constants whose values are dependent on the hypothesis $H_{0}$ or $H_{1}$. According to the system model given in Section II, this implies that the fusion center combines a linear output of correlators (i.e., constants) disturbed by AWGN.
Since the noise in the broadcast fusion channel is assumed to be of AWGN form, the linear combination in the fusion center yields another Gaussian random variable whose mean and variance are given in (16) and (17). Hence, the performance metric used for the system model is the following $\left\{P_{f}, P_{d}\right\}$ pair due to the aforementioned Gaussian assumption:

$$
\begin{aligned}
& P_{f}=Q\left[\frac{\gamma_{c}[n]-\Phi^{\prime}\|\mathbf{w}[n]\|_{1}}{\sqrt{\mathbf{w}^{T}[n] \mathbf{C o v}_{\mathbf{q}[\mathbf{n}]} \mathbf{w}[n]}}\right] \\
& P_{d}=Q\left[\frac{\gamma_{c}[n]-\Phi^{+}\|\mathbf{w}[n]\|_{\mathbf{1}}}{\sqrt{\mathbf{w}^{\mathbf{T}}[n] \mathbf{C o v}_{\mathbf{q}[\mathbf{n}]} \mathbf{w}[n]}}\right]
\end{aligned}
$$

where $P_{f}$ denotes the probability of false alarm, $P_{d}$ denotes the probability of detection, and $Q(\cdot)$ is the complementary cumulative distribution function, which calculates the tail probability of a zero-mean unit variance Gaussian random variable.

Here, note that different weight selection rules can be employed for different purposes, such as that described in [40] and [41]. In this paper, although the broadcast fusion channel is assumed to be of AWGN form, there is no restriction imposed on the channel (and on the type of propagation environment such as urban or suburban) between the sensors and the unknown signal source. Therefore, the system model can easily be extended to that in which an unknown signal $x(t)$ can be assumed to undergo shadowing, multipath fading, and Doppler spread (i.e., a mobile unknown signal source) as well.

As soon as $y_{c}[n]$ is calculated, an estimate of the test threshold for the corresponding time step $n$ should be calculated so that an error is obtained and the weights are updated accordingly. As discussed earlier, at each time step $n, \gamma_{c}[n]$ is calculated with the aid of both (18) and (19). Hence, the error value for the corresponding time step is calculated as

$$
e_{c}[n]=\gamma_{c}[n]-y_{c}[n] .
$$

For a fixed value of probability of false alarm $P_{f}$, one can obtain the corresponding threshold value $\gamma_{c}[n]$ from (18) as

$$
\gamma_{c}[n]=\Phi^{\prime}\|\mathbf{w}[n]\|_{\mathbf{1}}+Q^{-1}\left(P_{f}\right) \sqrt{\mathbf{w}^{\mathbf{T}}[n] \operatorname{Cov}_{\mathbf{q}[\mathbf{n}]} \mathbf{w}[n]}
$$

Along with the weight update equation to be presented, (21) provides self-adaptation of the weights in such a way that the statistics of $P_{f}$ are not affected by dynamic changes and drifts in the channel and/or the propagation environment. Considering the dynamic changes in the physical environment, to have a cooperative spectrum sensing system that maintains certain performance criteria while avoiding any assumptions on the physical world such as number of users, type of propagation medium, etc., one possible way is to incorporate a controlled feedback mechanism based on an error term $e_{c}[\cdot]$ to the decision making strategy. In the proposed online ADF framework, this is achieved by keeping the false alarm rate fixed, which in turn implies a constant value for the threshold $\gamma_{c}[n]$ in (21). Consequently, at each time step, the error value is evaluated as in (20) with respect to the test threshold. One of the main advantages of the proposed online cooperative spectrum 


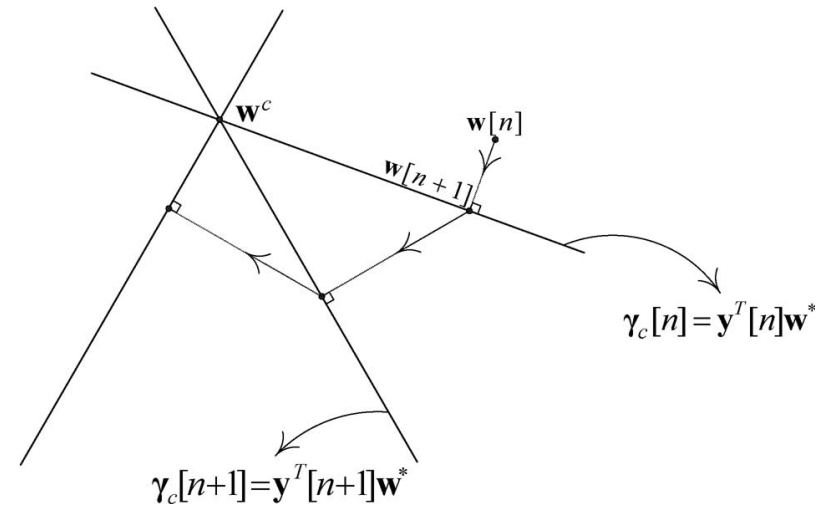

Fig. 2. Geometric interpretation. Weight vectors corresponding to correlator estimates at each time step are updated to satisfy the hyperplane equations defined by the test threshold $\gamma_{c}[n]$ and the output of the broadcast fusion channel $\mathbf{y}[n]$. Here, the lines represent hyperplanes in $\mathbb{R}^{M}$. If the channel and environmental conditions are kept fixed, iterated weights converge at the intersection of hyperplanes $\mathbf{w}^{\mathbf{c}}$, as discussed in [42].

sensing strategy is this feedback mechanism, as compared with other related methods like those discussed in [24]. The weights of the spectrum sensors yielding correlation estimates different than (same as) the test threshold are reduced (increased) iteratively at each time step, making it possible to keep the performance of sensing unaffected by the change in channel characteristics. It is worth mentioning at this point that the proposed algorithm is independent of any specific probability distribution on the data. In addition, as discussed earlier in this section, different values of $\Phi^{+}$for different environmental classes do not affect the analysis since (21) is independent of $\Phi^{+}$.

Set theoretic weight update algorithm: Ideally, the weighted sum of the received summary statistics of spectrum sensors should be equal to the test threshold $\gamma_{c}[n]$ at time instant $n$, i.e.,

$$
\gamma_{c}[n]=\mathbf{y}^{T}[n] \mathbf{w}[n]
$$

which represents a hyperplane in the $M$-dimensional space $\mathbf{w}[n] \in \mathbb{R}^{M}$. Hyperplanes are convex in $\mathbb{R}^{M}$. At time instant $n$, $\mathbf{y}^{T}[n] \mathbf{w}[n]$ may not be equal to $\gamma_{c}[n]$. The next set of weights are determined by projecting the current weight vector $\mathbf{w}[n]$ onto the hyperplane represented by (22). This process is geometrically depicted in Fig. 2 . The orthogonal projection $\mathbf{w}[n+$ 1] of the vector of weights $\mathbf{w}[n] \in \mathbb{R}^{M}$ onto the hyperplane $\gamma_{c}[n]=\mathbf{y}^{T}[n] \mathbf{w}[n]$ is the closest vector on the hyperplane to the vector $\mathbf{w}[n]$.

Let us formulate the problem as a minimization problem

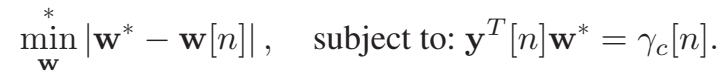

The solution can be obtained by using Lagrange multipliers

$$
\mathcal{L}=\sum_{i}\left(w_{i}[n]-w_{i}^{*}\right)^{2}+\lambda\left(\mathbf{y}^{T}[n] \mathbf{w}^{*}-\gamma_{c}[n]\right)
$$

taking partial derivatives with respect to $w_{i}^{*}$

$$
\frac{\partial \mathcal{L}}{\partial w_{i}^{*}}=2\left(w_{i}[n]-w_{i}^{*}\right)+\lambda y_{i}[n], \quad i=1, \ldots, M
$$

setting the result to zero

$$
2\left(w_{i}[n]-w_{i}^{*}\right)+\lambda y_{i}[n]=0, \quad i=1, \ldots, M
$$

and defining the next set of weights $\mathbf{w}[n+1]=\mathbf{w}^{*}$ as a set of $M$ equations is obtained

$$
\mathbf{w}[n+1]=\mathbf{w}[n]+\frac{\lambda}{2} \mathbf{y}[n] .
$$

The Lagrange multiplier $\lambda$ can be obtained from the condition equation

$$
\mathbf{y}^{T}[n] \mathbf{w}^{*}-\gamma_{c}[n]=0
$$

as follows:

$$
\lambda=2 \frac{\gamma_{c}[n]-y_{c}[n]}{\|\mathbf{y}[n]\|^{2}}=2 \frac{e_{c}[n]}{\|\mathbf{y}[n]\|^{2}}
$$

where the error term is given by (20). Plugging this into (27) yields

$$
\mathbf{w}[n+1]=\mathbf{w}[n]+\frac{e_{c}[n]}{\|\mathbf{y}[n]\|^{2}} \mathbf{y}[n] .
$$

Hence, the projection vector is calculated according to (30).

Whenever a new set of correlator estimates $\mathbf{V}_{\mathbf{U}}$ are generated by spectrum sensors, another hyperplane based on the new data values $\mathbf{y}[n]$ that arrived at the fusion center from the broadcast fusion channel is defined in $\mathbb{R}^{M}$, i.e.,

$$
\gamma_{c}[n+1]=\mathbf{y}^{T}[n+1] \mathbf{w}^{*} .
$$

This hyperplane will probably not be the same as the $\gamma_{c}[n]=$ $\mathbf{y}^{T}[n] \mathbf{w}[n]$ hyperplane, as shown in Fig. 2. The next set of weights $\mathbf{w}[n+2]$ are determined by projecting $\mathbf{w}[n+1]$ onto the hyperplane in (31). Iterated weights converge at the intersection of hyperplanes $\mathbf{w}^{\mathbf{c}}$, as stated in [42]. The rate of convergence can be adjusted by introducing a relaxation parameter $\mu$ to (30) as follows:

$$
\mathbf{w}[n+1]=\mathbf{w}[n]+\mu \frac{e_{c}[n]}{\|\mathbf{y}[n]\|^{2}} \mathbf{y}[n]
$$

where $0<\mu<2$ should be satisfied to guarantee convergence according to the POCS theory [43], [44].

The relaxation parameter has an important role in the convergence speed of POCS-based algorithms as this has been very well analyzed in the literature under the assumption of having a wide-sense stationary model [45, and references therein]. However, convergence may take infinitely long for the case where the hyperplanes in Fig. 2 do not intersect at all. This is true for most of the practical cases, and spectrum sensing is not an exception. In case the intersection of hyperplanes is an empty set, the updated weight vector simply satisfies the last hyperplane equation. In other words, it tracks the test threshold $\gamma_{c}[n]$ by assigning proper weights to individual spectrum sensors to maintain the same $P_{f}$ value under dynamically changing channel and propagation environment characteristics. Note that 
the proposed online decision fusion method does not need to wait for convergence to give a decision.

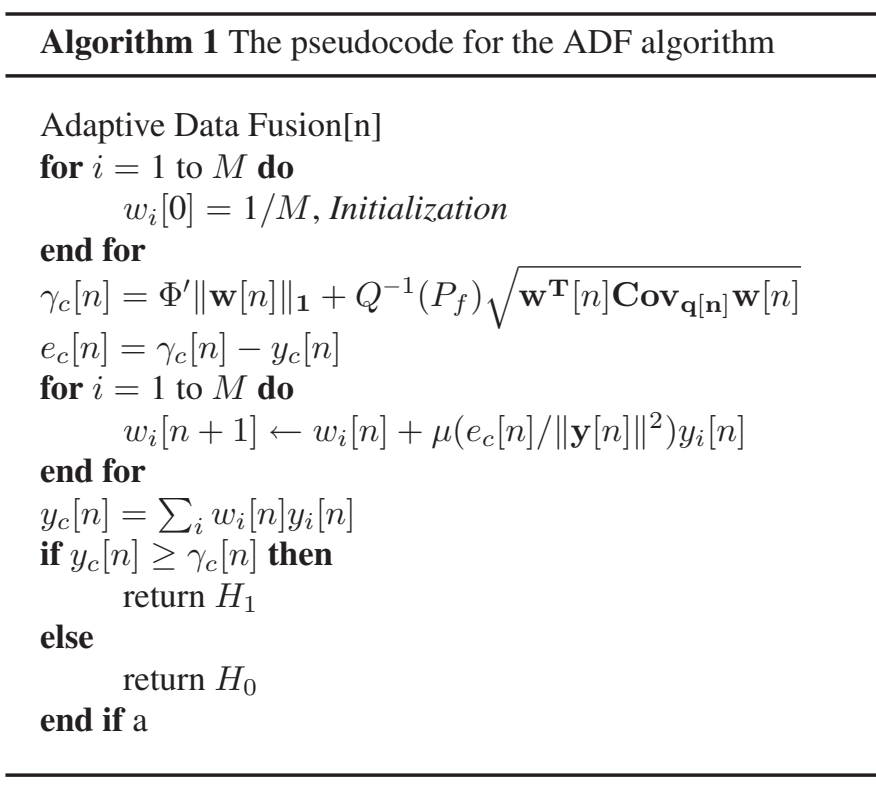

\section{NUMERICAL RESULTS AND DisCUSSIONS}

In the simulations, multiple spectrum sensors $(M>1)$ are assumed to employ their detectors individually to identify the presence of the same signal source and then to send their results to the fusion center, as depicted in Fig. 1. The spectrum sensors and fusion center are all assumed to be fixed, whereas the signal source, when it is actively transmitting, is assumed to be mobile with an average speed value of $v=10 \mathrm{~m} / \mathrm{s}$ and to operate on $2 \mathrm{GHz}$ within the same type of propagation environment. The fast-fading channels between the actively transmitting source and the fixed sensors are assumed to have a Rayleigh distributed amplitude with a Doppler spectrum that is of Jakes' type. Spatially correlated log-normal shadowing is applied to the signal with $\sigma_{G}=\{4.3,7.5\} \mathrm{dB}$ and $d_{\rho}=\{5.75,350\} \mathrm{m}$ for urban and suburban environments, respectively, as reported in [33]. These two particular environments are selected intentionally because the performance of the proposed method needs to be examined for physical environments that exhibit drastic differences in their propagation characteristics. The sampling frequency of the receiver is assumed to be fixed at $20 \mathrm{kHz}$ to satisfy the condition $f_{D} \ll f_{s}$. The effective averaging time of the low-pass integrate-and-dump filter is set to $0.05 \mathrm{~ms}$, whereas the total sensing time is set to $1 \mathrm{~ms}$. The impact of path loss is neglected under hypothesis $H_{1}$ driven by the fact that sensors generate their results in a very short period of time, which leads to a very short displacement that the mobile traverses within that period. In light of this, spectrum sensors are assumed to observe the same SNR, which is selected to be $3.5 \mathrm{~dB}$, reflecting a relatively strong presence when the source is actively transmitting.

To see the beneficial impact of cooperation for the given settings, Fig. 3 should be examined first. It is clear in the figure that the increase in the number of spectrum sensors contributing to the decision making mechanism improves the overall system performance drastically, as expected. As will

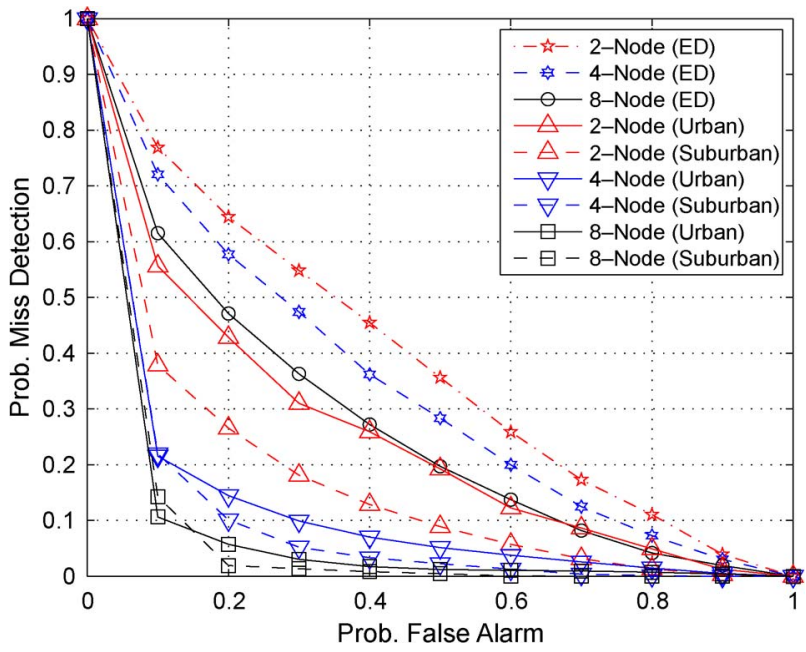

Fig. 3. Performance comparison for the adaptive fusion decision mechanism for different numbers of spectrum sensors that employ the conventional energy detector [labeled with "(ED)" in the plots] and second-order detector in different scenarios

be shown subsequently, this behavior is maintained by the proposed method regardless of the type of detector employed in the sensors or of the propagation environment considered.

The performance of the proposed method when a drift in concepts takes place, such as a change in propagation environment from suburban to urban, needs to be investigated as well. For this purpose, the proposed method is tested for an urban environment scenario, and the results are presented in Fig. 3 along with the suburban environment results for the sake of comparison. The overall performance of the proposed method exhibits the same behavior in the urban scenario as it does in the suburban scenario. However, it is important to note that the proposed method performs slightly better in the suburban scenario than in the urban scenario. This stems from the fact that the second-order statistical detectors employed in sensors can take advantage of the large decorrelation distance that shapes the shadowing process and therefore performs better. In this regard, although the suburban environment has greater standard deviation, its larger decorrelation distance dominates and yields better results.

For comparison purposes, the performance of the proposed method can be examined by replacing each second-order detector in sensors with the conventional energy detector while keeping all of the remaining settings intact. Results for such a scenario are also shown in Fig. 3. As can be seen from the plots, the energy detector-based adaptive fusion mechanism performs worse compared with the second-order detector-based adaptive fusion framework. This is because the second-order detectors are able to extract the correlation information from the received signal although it undergoes both fast and slow fading. On the other hand, the energy detector relies solely on the power statistics that can be severely degraded by shadowing and deep fast-fading scenarios; therefore, it yields a weaker performance. Here, it is important to state that the second-order detector used in sensors can be transformed into the conventional energy detector by deactivating several blocks, which can be considered within the software-defined radio concept. 


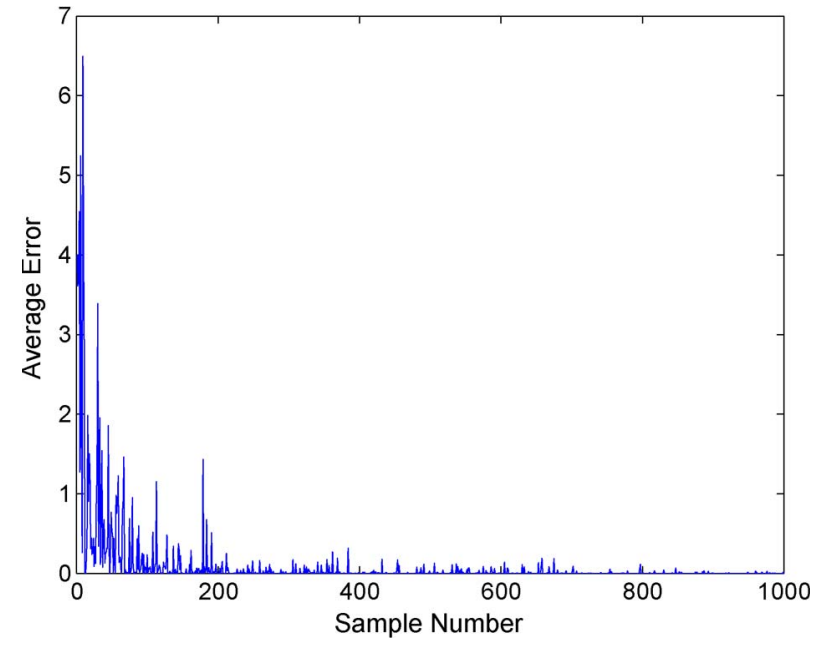

Fig. 4. Convergence of Algorithm 1 for $0.1<P_{f}$.

Although it is not required by the online ADF framework, in accordance with the POCS theory, Algorithm 1 is shown to converge by exhibiting a decrease in the average error values over time in Fig. 4. Note in Fig. 4 that the channel conditions between the source and the sensors are kept the same throughout the convergence period for illustrative purposes. The average error is evaluated for $P_{f}$ values greater than 0.1 . The convergence rate can be adjusted by changing the relaxation parameter $\mu$ in (32). It is important to state that for smaller values of $P_{f}$, which correspond to more demanding cases, the convergence rate will be smaller accordingly, resulting in longer durations to achieve similar average error values.

As stated earlier, the energy detector is a special case of the second-order detector. In addition, theoretically speaking, the output of the energy detector is unbounded, whereas the output of the second-order detector, namely, $V_{U}$, is bounded. This implies that employing energy detection at sensors will challenge the decision fusion mechanism and provide a practical upper bound in terms of $\left(P_{f}, P_{d}\right)$ pair, as shown in Fig. 3. Furthermore, as can be observed in (5), many critical practical concerns such as imperfect carrier and phase recovery are disregarded in the analysis. Hence, the robustness of the proposed method is tested with a more practical scenario that includes the physical implementation of the system model [28], [29]. In [28] and [29], the robustness of the proposed method is tested, including the following three aspects. First of all, real wireless signals are generated and captured over the air. Second, the fusion center in the experimental setup is exposed to receiver impairments such as inherent low-pass filtering, imperfect carrier, and phase recovery. Third, based on the results plotted in Fig. 3, the energy detector is employed at the sensors to challenge the overall performance of the method proposed further. The results in [28] and [29] show that the decision fusion mechanism is robust under practical conditions, including the impact of real wireless propagation environment and receiver impairments. As reported and discussed in [28] and [29], the correlated input to the ADF scheme has a positive impact on the performance of cooperative spectrum sensing by yielding lower error values, which in turn results in fast convergence of weights.

\section{CONCLUSION}

An online adaptive cooperative spectrum sensing scheme based on the POCS theory has been proposed to maintain the performance and reliability of sensing under dynamically changing channel and environmental conditions, such as correlated shadowing and fast fading. Each collaborating sensor performs a second-order analysis on the received signal that is assumed to be transmitted by a single mobile source. The contributions of this paper are threefold. First, the main propagation mechanisms including fast- and slow-fading phenomena that affect the mobile radio channel are incorporated into the spectrum sensing. Second, the received signal by the sensors are processed with second-order detectors that provide the fusion center with constant output. Finally, an online ADF scheme to be deployed in a cooperative spectrum sensing framework is introduced.

Results show that the proposed method improves the performance of the second-order detector with the aid of cooperation. In addition, comparative analysis reveals that the adaptive fusion mechanism supported by second-order detector output exhibits superior performance over conventional energy-based spectrum detectors.

\section{APPENDIX \\ ProOF OF Proposition 1}

Under the hypothesis $H_{0}$, note that $T \rightarrow \infty$ and $T_{A}=$ 0 imply that there is infinite support with no low-pass filtering; therefore, in (7), $N_{F}(t)$ immediately degenerates to $n(t)$. In that case, the input to the logarithm operator has a chi-square distribution with 2 degrees of freedom $\left(\chi^{2}(2)\right)$. However, since the input to the logarithm operator is generated by the squaring device, one can take advantage of the equality

$$
\ln \left(|n(t)|^{2}\right)=2 \ln (|n(t)|) .
$$

This way, the input-output relationship for the logarithm operator can be expressed in terms of the Rayleigh distribution, which provides analytical tractability in the subsequent steps. Because $\mathcal{Y}(t)=|n(t)|$ is Rayleigh distributed, its probability density function (pdf) is given by

$$
p_{\mathcal{Y}}(y)= \begin{cases}\frac{y}{\alpha} e^{\left(-\frac{y^{2}}{2 \alpha}\right)}, & 0 \leq y \\ 0, & y<0\end{cases}
$$

where $\alpha$ is the mode parameter of the distribution satisfying $\mu y=\alpha \sqrt{(\pi / 2)}$, with $\mu y$ being the mean of the pdf of $\mathcal{Y}(t)$ and $\alpha=\sqrt{\sigma_{N}^{2} / 2}$. Therefore, the output of the logarithm, for example, $\mathfrak{X}(t)$, forms a time series that is composed of logRayleigh distributed values

$$
\mathfrak{X}(t)=\ln (\mathcal{Y}(t))=\ln (|n(t)|)
$$

with the following pdf:

$$
p_{\mathfrak{X}}(\mathfrak{x})=\frac{e^{2 \mathfrak{x}}}{\alpha} \exp \left(-\frac{e^{2 \mathfrak{x}}}{2 \alpha}\right)
$$


for all $\mathfrak{x} \in \mathbb{R}$. Since the output of the correlator is difficult to express in closed form when the input is solely noise, let

$$
r^{\prime}(t)=\lim _{\rightarrow 0} A \cos \left(2 \pi f_{A} t+\phi_{A}\right)+n(t)
$$

where $A, f_{A}$, and $\phi_{A}$ are some arbitrary amplitude, frequency, and phase values, respectively. Note that (36) is equivalent to the hypothesis $H_{0}$ in the limiting sense. If $r^{\prime}(t)$ follows through steps (7)-(10), then the output of the correlator is given by [46]

$$
\begin{aligned}
\Psi(\tau)= & \sum_{\substack{i=1 \\
(i+l \text { even })}}^{\infty} \varphi_{N}^{k}(\tau) \sum_{l=1}^{i}\left(\begin{array}{c}
(i+l) / 2-1 \\
(i-l) / 2
\end{array}\right) \Upsilon^{m} \\
& \times{ }_{1} F_{1}^{2}((i+l) / 2 ; l+1 ;-\Upsilon) / l ! l(i+l) \\
& +\frac{1}{4} \sum_{i=1}^{\infty}{ }_{1} F_{1}^{2}(i ; 1 ;-\Upsilon) \varphi_{N}^{2 k}(\tau)+\left(\ln (A)+\frac{1}{2} \mathfrak{E}_{1}(\Upsilon)\right)^{2}
\end{aligned}
$$

where $\varphi_{N}(\cdot)$ is the normalized autocorrelation estimate of the quadrature components (i.e., $n_{Q}(\cdot)$ ) of $n(t), \Upsilon$ is the SNR and defined to be $\Upsilon \triangleq A^{2} /\left(2 \sigma_{N}^{2}\right),{ }_{1} F_{1}^{2}(\cdot ; \cdot ; \cdot)$ is the confluent hypergeometric function, and $\mathfrak{E}_{1}(\cdot)$ is the exponential integral [47]. Since the purpose is to obtain the characteristics of the noise-only process, one can consider (37) by expanding $\mathfrak{E}_{1}(\cdot)$ into a power series for $A \rightarrow 0$ (or equivalently for $\Upsilon \rightarrow 0$ ). This allows one to see that (37) is dominated by the cross-noise terms as $\Upsilon$ diminishes and can be expressed after some manipulations as

$$
R_{\mathfrak{X}}(\tau) \triangleq \lim _{\Upsilon \rightarrow 0} \Psi(\tau) \cong \frac{1}{4} \sum_{i=1}^{\infty} \frac{\varphi_{N}^{2 k}(\tau)}{i^{2}}+\Phi\left(\sigma_{N}\right)
$$

where $\Phi\left(\sigma_{N}\right)$ represents a constant that depends on the noise variance $\sigma_{N}^{2}$. Since $\Phi\left(\sigma_{N}\right)$ is a constant, one can readily calculate the variance of $\mathfrak{X}(t)$ by setting $\tau=0$ and ignoring $\Phi\left(\sigma_{N}\right)$ as

$$
\sigma_{\mathfrak{X}}^{2}=\frac{1}{4} \sum_{i=1}^{\infty} \frac{1}{i^{2}}=\frac{\pi^{2}}{24} .
$$

Recalling that $\mathfrak{X}(t)$ is a nonzero mean process (i.e., $\mu_{\mathfrak{X}} \neq$ $0)$ due to the nonlinear transformation applied, $\Phi\left(\sigma_{N}\right)=\mu_{\mathfrak{X}}^{2}$ holds in (38) since $R_{\mathfrak{A}}(\tau)=E\left\{\mathfrak{A}(t) \mathfrak{A}^{*}(t+\tau)\right\}$ and $R_{\mathfrak{A}}(0)=$ $\sigma_{\mathfrak{A}}^{2}+\mu_{\mathfrak{A}}^{2}$ for any stationary stochastic process $\mathfrak{A}(t)$ with $\mu_{\mathfrak{A}} \neq 0$.

Then, by assuming $\sigma_{N}$ to be unity for the sake of simplicity, $\mu_{\mathfrak{X}}$ can be calculated via (33) and (35)-(38) as

$$
\mu_{\mathfrak{X}}=\ln (\alpha)+\frac{\ln (2)-\gamma}{2}=\ln \left(\frac{1}{\sqrt{2}}\right)+\frac{\ln (2)-\gamma}{2}=-\frac{\gamma}{2}
$$

where $\gamma$ is the Euler-Mascheroni (or sometimes referred to solely as Euler's) constant and given by $\gamma=$ $-\int_{0}^{\infty} \ln (u) e^{-u} d u{ }^{4}$ In (38), it is clear that at larger delays

\footnotetext{
${ }^{4}$ The first five digits of $\gamma$ in decimal form are $\gamma \cong 0.57721 \ldots$
}

(lags) $\tau$, the autocorrelation estimates exhibit an asymptotic behavior and converge $\Phi\left(\sigma_{N}\right)$, which is a function of noise variance. However, it is desired that the method proposed is independent of noise variance $\sigma_{N}^{2}$. Thus, normalizing the autocorrelation estimates with the signal power (i.e., with the value at $\tau=0$ ) will yield the following constant ${ }^{5}$ :

$$
\Phi^{\prime}=\frac{\Phi\left(\sigma_{N}\right)}{R_{\mathfrak{X}}(0)}=\frac{\mu_{\mathfrak{X}}^{2}}{\mu_{\mathfrak{X}}^{2}+\sigma_{\mathfrak{X}}^{2}} .
$$

Finally, if (40) is placed in (41), the following is obtained:

$$
\Phi^{\prime}=\frac{\gamma^{2}}{\gamma^{2}+\pi^{2} / 6}
$$

which concludes the proof.

\section{REFERENCES}

[1] J. Mitola, "Cognitive radio architecture evolution," Proc. IEEE, vol. 97, no. 4, pp. 626-641, Apr. 2009.

[2] Y. Hur, J. Park, W. Woo, J. S. Lee, K. Lim, C.-H. Lee, H. S. Kim, and J. Laskar, "A cognitive radio (CR) system employing a dual-stage spectrum sensing technique: a multi-resolution spectrum sensing (MRSS) and a temporal signature detection (TSD) technique," in Proc. IEEE GLOBECOM, San Francisco, CA, Nov. 27-Dec. 1 2006, pp. 1-5.

[3] W.-Y. Lee and I. F. Akyildiz, "Optimal spectrum sensing framework for cognitive radio networks," IEEE Trans. Wireless Commun., vol. 7, no. 10, pp. 3845-3857, Oct. 2008.

[4] H. Urkowitz, "Energy detection of unknown deterministic signals," Proc. IEEE, vol. 55, no. 4, pp. 523-531, Apr. 1967.

[5] A. Sonnenschein and P. M. Fishman, "Radiometric detection of spreadspectrum signals in noise of uncertain power," IEEE Trans. Aerosp. Electron. Syst., vol. 28, no. 3, pp. 654-660, Jul. 1992.

[6] H. Tang, "Some physical layer issues of wide-band cognitive radio systems," in Proc. 1st IEEE Int. Symp. New Frontiers DySPAN, Baltimore, MD, Nov. 8-11, 2005, pp. 151-159.

[7] R. A. Dillard, "Detectability of spread-spectrum signals," IEEE Trans. Aerosp. Electron. Syst., vol. AES-15, no. 4, pp. 526-537, Jul. 1979.

[8] J. G. Proakis, Digital Communications. New York: McGraw-Hill, 2001.

[9] N. F. Krasner, "Optimal detection of digitally modulated signals," IEEE Trans. Commun., vol. COM-30, no. 5, pp. 885-895, May 1982.

[10] A. Sahai, R. Tandra, S. M. Mishra, and N. Hoven, "Fundamental design tradeoffs in cognitive radio systems," in Proc. 1st Int. Workshop TAPAS, Boston, MA, Aug. 5, 2006, pp. 1-9.

[11] M. Öner and F. Jondral, "Cyclostationarity based air interface recognition for software radio systems," in Proc. IEEE Radio Wireless Conf., Atlanta, GA, Sep. 19-22, 2004, pp. 263-266.

[12] A. Fehske, J. Gaeddert, and J. H. Reed, "A new approach to signal classification using spectral correlation and neural networks," in Proc. 1st IEEE Int. Symp. New Frontiers DySPAN, Baltimore, MD, Nov. 8-11, 2005, pp. 144-150.

[13] W. A. Gardner, "Signal interception: a unifying theoretical framework for feature detection," IEEE Trans. Commun., vol. 36, no. 8, pp. 897-906, Aug. 1988.

[14] T. Ikuma and M. Naraghi-Pour, "Autocorrelation-based spectrum sensing algorithms for cognitive radios," in Proc. 17th ICCCN, St. Thomas, U.S. Virgin Islands, Aug. 3-7, 2008, pp. 1-6.

[15] R. K. Sharma and J. W. Wallace, "Improved spectrum sensing by utilizing signal autocorrelation," in Proc. IEEE 69th VTC, Barcelona, Spain, Apr. 26-29, 2009, pp. 1-5.

[16] K. A. Qaraqe and S. Yarkan, "A second-order statistical method for spectrum sensing in correlated shadowing and fading environments," in Proc. 21st Аnnu. IEEE Int. Symp. PIMRC, Istanbul, Turkey, Sep. 26-29, 2010, pp. 780-785.

\footnotetext{
${ }^{5}$ When $\sigma_{N}$ is unity: $\Phi^{\prime}=\left(\gamma^{2} / \gamma^{2}+\pi^{2} / 6\right) \cong 0.16843 \ldots$ for the first five significant digits in decimal.
} 
[17] H. Arslan and T. Yücek, "Spectrum sensing for cognitive radio applications," in Cognitive Radio, Software Defined Radio, and Adaptive Wireless Systems (Signals and Communication Technology). New York: SpringerVerlag, 2007.

[18] T. Yücek and H. Arslan, "Spectrum characterization for opportunistic cognitive radio systems," in Proc. IEEE Conf. MILCOM, Piscataway, NJ, 2006, pp. 1805-1810.

[19] T. Yücek and H. Arslan, "A survey of spectrum sensing algorithms for cognitive radio applications," IEEE Commun. Surveys Tuts., vol. 11, no. 1, pp. 116-130, First Quarter, 2009.

[20] Y. Zeng, Y.-C. Liang, A. T. Hoang, and R. Zhang, "A review on spectrum sensing for cognitive radio: challenges and solutions," EURASIP J. Adv. Signal Process., vol. 2010, pp. 1-15, Jan. 2010.

[21] L. Chen, J. Wang, and S. Li, "An adaptive cooperative spectrum sensing scheme based on the optimal data fusion rule," in Proc. 4th ISWCS, Trondheim, Norway, Oct. 17-19, 2007, pp. 582-586.

[22] J. Myung, K. Lee, J. Kang, and J. Kang, "Adaptive cooperative spectrumsensing scheme for cognitive radio system," in Proc. IEEE 72nd VTC, Ottawa, ON, Canada, Sep. 6-9, 2010, pp. 1-5.

[23] I. F. Akyildiz, W.-Y. Lee, M. C. Vuran, and S. Mohanty, "Next generation/dynamic spectrum access/cognitive radio wireless networks: A survey," Comput. Netw., vol. 50, no. 13, pp. 2127-2159, Sep. 2006.

[24] Z. Quan, S. Cui, and A. H. Sayed, "Optimal linear cooperation for spectrum sensing in cognitive radio networks," IEEE J. Sel. Topics Signal Process., vol. 2, no. 1, pp. 28-40, Feb. 2008.

[25] K. B. Letaief and W. Zhang, "Cooperative communications for cognitive radio networks," Proc. IEEE, vol. 97, no. 5, pp. 878-893, May 2009

[26] I. F. Akyildiz, B. F. Lo, and R. Balakrishnan, "Cooperative spectrum sensing in cognitive radio networks: a survey," Phys. Commun., vol. 4, no. 1, pp. 40-62, Mar. 2011.

[27] B. U. Toreyin, "Fire detection algorithms using multimodal signal and image analysis," Ph.D. dissertation, Bilkent Univ., Ankara, Turkey, Jan. 2009.

[28] S. Yarkan, B. U. Töreyin, K. A. Qaraqe, and E. A. Cetin, "An experimental validation of an online adaptive cooperation scheme for spectrum sensing," in Proc. 18th ICT, Ayia Napa, Cyprus, May 8-11, 2011, pp. 226-231.

[29] S. Yarkan, K. Qaraqe, B. U. Töreyin, and E. A. Cetin, "An experimental setup for performance analysis of an online adaptive cooperative spectrum sensing scheme for both in- phase and quadrature branches," in Proc. 74th IEEE VTC, San Francisco, CA, Sep. 5-8, 2011, pp. 1-5.

[30] G. L. Stüber, Principles of Mobile Communications, 4th ed. Norwell, MA: Kluwer, 1996.

[31] M. Pätzold, U. Killat, and F. Laue, "A deterministic digital simulation model for Suzuki processes with application to a shadowed Rayleigh land mobile radio channel," IEEE Trans. Veh. Technol., vol. 45, no. 2, pp. 318331, May 1996

[32] X. Cai and G. B. Giannakis, "A two-dimensional channel simulation model for shadowing processes," IEEE Trans. Veh. Technol., vol. 52, no. 6, pp. 1558-1567, Nov. 2003.

[33] M. Gudmundson, "Correlation model for shadow fading in mobile radio systems," Electron. Lett., vol. 27, no. 23, pp. 2145-2146, Nov. 1991.

[34] C. Oestges, N. Czink, B. Bandemer, P. Castiglione, F. Kaltenberger, and A. J. Paulraj, "Experimental characterization and modeling of outdoorto-indoor and indoor-to-indoor distributed channels," IEEE Trans. Veh. Technol., vol. 59, no. 5, pp. 2253-2265, Jun. 2010.

[35] J. Ojala, R. Bohnke, and M. Uno, "On the propagation characteristics of the $5 \mathrm{GHz}$ rooftop-to-rooftop meshed network," in Proc. IST Mobile Wireless Telecommun. Summit, Thessaloniki, Greece, Jun. 17-19, 2002.

[36] W. Fischer, Digital Television: A Practical Guide for Engineers. New York: Springer-Verlag, 2004.

[37] T. Kratochvil, "DVB-T/H laboratory transmission using fading channel profiles," in Proc. 15th IWSSIP, Bratislava, Slovak Republic, Jun. 25-28, 2008, pp. 343-346.

[38] R. D. Bari, M. Bard, A. Arrinda, P. Ditto, J. Cosmas, K. K. Loo, and R. Nilavalan, "Rooftop and indoor reception with transmit diversity applied to DVB-T networks: a long term measurement campaign," in Proc. IEEE Int. Symp. BMSB, Bilbao, Spain, May 13-15, 2009, pp. 1-6.

[39] J. Ventura-Traveset, G. Caire, E. Biglieri, and G. Taricco, "Impact of diversity reception on fading channels with coded modulation-Part I: Coherent detection," IEEE Trans. Commun., vol. 45, no. 5, pp. 563-572, May 1997.
[40] K. Zeng, P. Pawelczak, and D. Cabric, "Reputation-based cooperative spectrum sensing with trusted node assistance," IEEE Commun. Lett., vol. 14, no. 3, pp. 226-228, Mar. 2010.

[41] X. Zheng, J. Wang, Q. Wu, and J. Chen, "Cooperative spectrum sensing algorithm based on Dempster-Shafer theory," in Proc. 11th IEEE Singapore ICCS, 2008, pp. 218-221.

[42] P. L. Combettes, "The foundations of set theoretic estimation," Proc. IEEE, vol. 81, no. 2, pp. 182-208, Feb. 1993.

[43] D. C. Youla and H. Webb, "Image restoration by the method of convex projections, Part I-theory," IEEE Trans. Med. Imag., vol. MI-1, no. 2, pp. 81-94, Oct. 1982.

[44] U. Niesen, D. Shah, and G. W. Wornell, "Adaptive alternating minimization algorithms," IEEE Trans. Inf. Theory, vol. 55, no. 3, pp. 1423-1429, Mar. 2009.

[45] S. Theodoridis, K. Slavakis, and I. Yamada, "Adaptive learning in a world of projections," IEEE Signal Process. Mag., vol. 28, no. 1, pp. 97-123, Jan. 2011.

[46] J. A. Roberts, "Logarithmic detection with postdetection filtering," IEEE Trans. Aerosp. Electron. Syst., vol. AES-14, pp. 35-43, Jan. 1978.

[47] M. Abramowitz and I. A. Stegun, Handbook of Mathematical Functions: With Formulas, Graphs, and Mathematical Tables. New York: Dover, 1965.

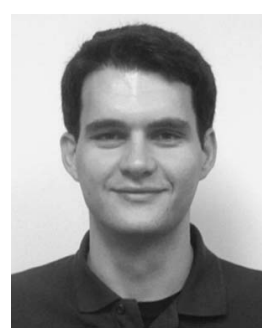

Serhan Yarkan received the B.S. and M.Sc. degrees in computer science from Istanbul University, Istanbul, Turkey, in 2001 and 2003, respectively, and the $\mathrm{Ph} . \mathrm{D}$. degree from the University of South Florida, Tampa, in 2009 .

From 2010 to November 2011, he was a Postdoctoral Research Associate with the Department of Computer and Electrical Engineering, Texas A\&M University, College Station. Since December 2011, he has been an Assistant Professor with the Department of Electrical and Electronics Engineering, Istanbul Commerce University. His research interests include statistical signal processing, cognitive radio, wireless propagation channel modeling, cross-layer adaptation and optimization, and interference management in next-generation wireless networks.

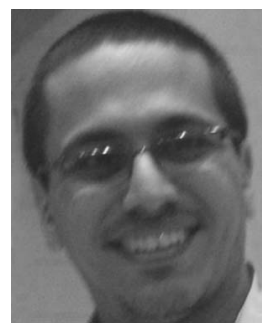

Behçet Uğur Töreyin received the B.S. degree in electrical and electronics engineering from Middle East Technical University, Ankara, Turkey, and the M.S. and Ph.D. degrees in electrical and electronics engineering from Bilkent University, Ankara.

Between 2009 and 2011, he was a Postdoctoral Research Associate with the Robotic Sensor Networks Lab, University of Minnesota, Minneapolis, and the Wireless Research Lab, Texas A\&M University at Qatar, Doha, Qatar, respectively. He is currently an Assistant Professor with Çankaya Uni- 


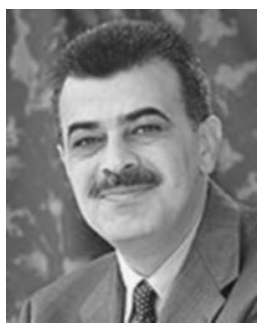

Khalid A. Qaraqe (M'97-S'00) was born in Bethlehem. He received the B.S. degree (with honors) in electronics engineering from the University of Technology, Baghdad, Iraq, in 1986, the M.S. degree in electronics engineering from the University of Jordan, Amman, Jordan, in 1989, and the Ph.D. degree in electronics engineering from Texas A\&M University, College Station, in 1997.

From 1989 to 2004 , he held a variety of positions with many companies, and he has over 12 years of experience in the telecommunication industry. He has worked for Qualcomm, Enad Design Systems, Cadence Design Systems/Tality Corporation, STC, SBC, and Ericsson. He has worked on numerous Global System for Mobile Communications, code division-multiple access (CDMA), and wideband CDMA projects and has experience in product development, design, deployments, testing, and integration. Since July 2004, he has been with the Department of Electrical Engineering, Texas A\&M University at Qatar, Doha, Qatar, where he is currently a Professor. His research interests include communication theory and its application to design and performance, analysis of cellular systems, and indoor communication systems. Particular interests are in the development of third-generation Universal Mobile Telecommunications Systems, cognitive radio systems, broadband wireless communications, and diversity techniques.

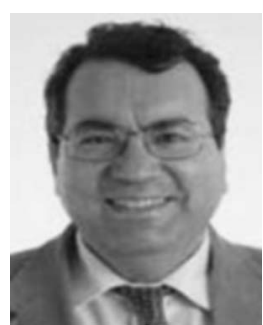

A. Enis Çetin (F'10) received the B.Sc. degree in electrical engineering from the Middle East Technical University, Ankara, Turkey, and the M.S.E. and Ph.D. degrees in systems engineering from the University of Pennsylvania, Philadelphia.

Between 1987 and 1989, he was an Assistant Professor of electrical engineering with the University of Toronto, Toronto, ON, Canada. Since 1989, he has been with Bilkent University, Ankara, where he is currently a Full Professor. He was with the University of Minnesota, Minneapolis, from 1996 to 1997, and Ryerson University, Toronto, from 2009 to 2010.

Dr. Çetin was an Associate Editor of the IEEE TRANSACTIONS ON IMAGE PROCESSING between 1999 and 2003 and a member of the SPTM technical committee of the IEEE Signal Processing Society. He is currently on the editorial board of EURASIP Journal of Advances in Signal Processing and Machine Vision and Applications. He was one of the founders of the Turkish Chapter of the IEEE Signal Processing Society in 1991. 